\title{
Advances in cell lineage reprogramming
}

\author{
ZHOU JunNian, YUE Wen \& PEI XueTao*
}

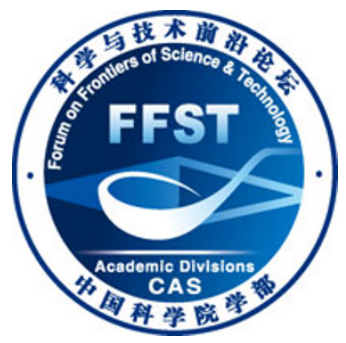

Stem Cell and Regenerative Medicine Lab, Beijing Institute of Transfusion Medicine, Beijing 100850, China

Received July 18, 2012; accepted January 9, 2013

\begin{abstract}
As a milestone breakthrough of stem cell and regenerative medicine in recent years, somatic cell reprogramming has opened up new applications of regenerative medicine by breaking through the ethical shackles of embryonic stem cells. However, induced pluripotent stem (iPS) cells are prepared with a complicated protocol that results in a low reprogramming rate. To obtain differentiated target cells, iPS cells and embryonic stem cells still need to be induced using step-by-step procedures. The safety of induced target cells from iPS cells is currently a further concerning matter. More broadly conceived is lineage reprogramming that has been investigated since 1987. Adult stem cell plasticity, which triggered interest in stem cell research at the end of the last century, can also be included in the scope of lineage reprogramming. With the promotion of iPS cell research, lineage reprogramming is now considered as one of the most promising fields in regenerative medicine, will hopefully lead to customized, personalized therapeutic options for patients in the future.
\end{abstract}

regenerative medicine, lineage reprogramming, transdifferentiation, dedifferentiation, transdetemination

Citation: Zhou J N, Yue W, Pei X T. Advances in cell lineage reprogramming. Sci China Life Sci, 2013, 56: 228-233, doi: 10.1007/s11427-013-4447-7

The word "programme" stands for a list of instructions written in a programming language, which controls the behavior of a machine or computer. The programming language is known as a computer language for computers, while in cells, it is an epigenetic language in which programme means a series of normal developmental and differentiation processes arranged in an orderly manner. "Reprogramme" is the reversal of programming. It refers to erasure and remodeling of the functional state of the genome, resulting in conversion of a differentiated cell to an immature cell. Reprogramming can be divided into two types: pluripotent and lineage reprogramming. Of the two, the former includes nuclear transfer, cell fusion, iPS cell reprogramming and long-term in vitro cell culture [1]. The latter refers to directly converting specialized cells (or progenitor cells) from one lineage to another without conversion to the iPS cell stage, including transdifferentiation,

*Corresponding author (email: peixt@nic.bmi.ac.cn) dedifferentiation, transdetermination, and metaplasia [2].

In the review of the history of reprogramming, we found that the development of lineage reprogramming occurred earlier than iPS cell establishment in 2006 [3]. In 1987, Davis et al. successfully reprogrammed fibroblasts to muscle cells by forced expression of the transcription factor MyoD [4]. In 2004, Xie et al. achieved reprogramming of B cells into macrophages by forced expression of the transcription factor $\mathrm{C} / \mathrm{EBP} \alpha$ [5]. In an interview after winning the Nobel Prize, Yamanaka said that he was inspired by the discovery of Davis in 1987. Therefore, the studies of lineage reprogramming laid the foundation for the emergence of iPS cells. This article will systematically review the research progress of lineage reprogramming since 1999 when Petersen et al. reported a bone marrow source of hepatocytes [6].

\section{Transdifferentiation}

Transdifferentiation refers to direct fate switching between 
two distinct cell types without passing through the pluripotency stage, which is also called lineage switching or lineage conversion [7].

Studies of adult stem cell plasticity, including bone marrow-derived hematopoietic stem cells (HSCs) and mesenchymal stem cells (MSCs), brought about worldwide enthusiasm for stem cells in biological and medical fields. As the first well studied and recognized stem cell types, HSCs had been the focus of most research concerning adult stem cell plasticity. In addition to hematopoietic reconstitution and a tri-lineage differentiation potential including osteoblasts, adipocytes and chondroblasts, many studies have demonstrated that HSCs and MSCs can be induced into various types of functional cells including hepatocytes, skeletal muscle cells, cardiac cells, endothelial cells, and neural cells $[8,9]$.

During this time, most researchers hypothesized a mechanism of "transdifferentiation". It should be noted that the concept of "transdifferentiation", in which the cells are adult stem cells rather than differentiated cells, is different from transdifferentiation described in this review. Here, we categorize "transdifferentiation" into transdetermination that will be discussed in detail below.

Since 2006, transdifferentiation strategy-based cell conversions from one mature cell type to another target cell type have been achieved by forced expression of transcription factors essential for development of the lineage (Table
1). Such achievements have been extended from neural cells to other tissues including blood, heart, and liver. Fibroblasts have been used as starting cells in most of the studies, because of their accessibility. Several conclusions can be drawn from Table 1: (i) In principle, transdifferentiation is easier between two closely related cell types than it is for more distantly related cell types. For example, only a single transcription factor $(\mathrm{C} / \mathrm{EBP} \alpha$ or $\mathrm{C} / \mathrm{EBP} \beta)$ is needed for cell conversion from $\mathrm{B}$ cells to macrophages [5], whereas both PU.1 and $\mathrm{C} / \mathrm{EBP} \alpha$ are necessary for conversion of fibroblasts to macrophages [10]. (ii) Transdifferentiation is easier in mouse cells than that in human cells in terms of conversion efficiency and the required number of transcription factors. For example, only three transcription factors (Ascl1, Brn2, and Myt11) are needed for cell conversion from fibroblasts to neural cells [13], whereas the same conversion in human cells is unsuccessful without the addition of another transcription factor, namely NeuroD1 [14], or one of Lmx1a and FoxA2 [15].

Most of the starting cell types and associated final target cell types in transdifferentiation originate from the same germ layer summarized in Table 1. Based on the same strategy, transdifferentiation has achieved conversion of fibroblasts to hepatocytes $[18,19]$, and hepatocytes to neural cells [20], which are the most clear transdifferentiation events that cross germ layers. These findings are advancing the success of regenerative medicine.

Table 1 Transdifferentiation ${ }^{\mathrm{a})}$

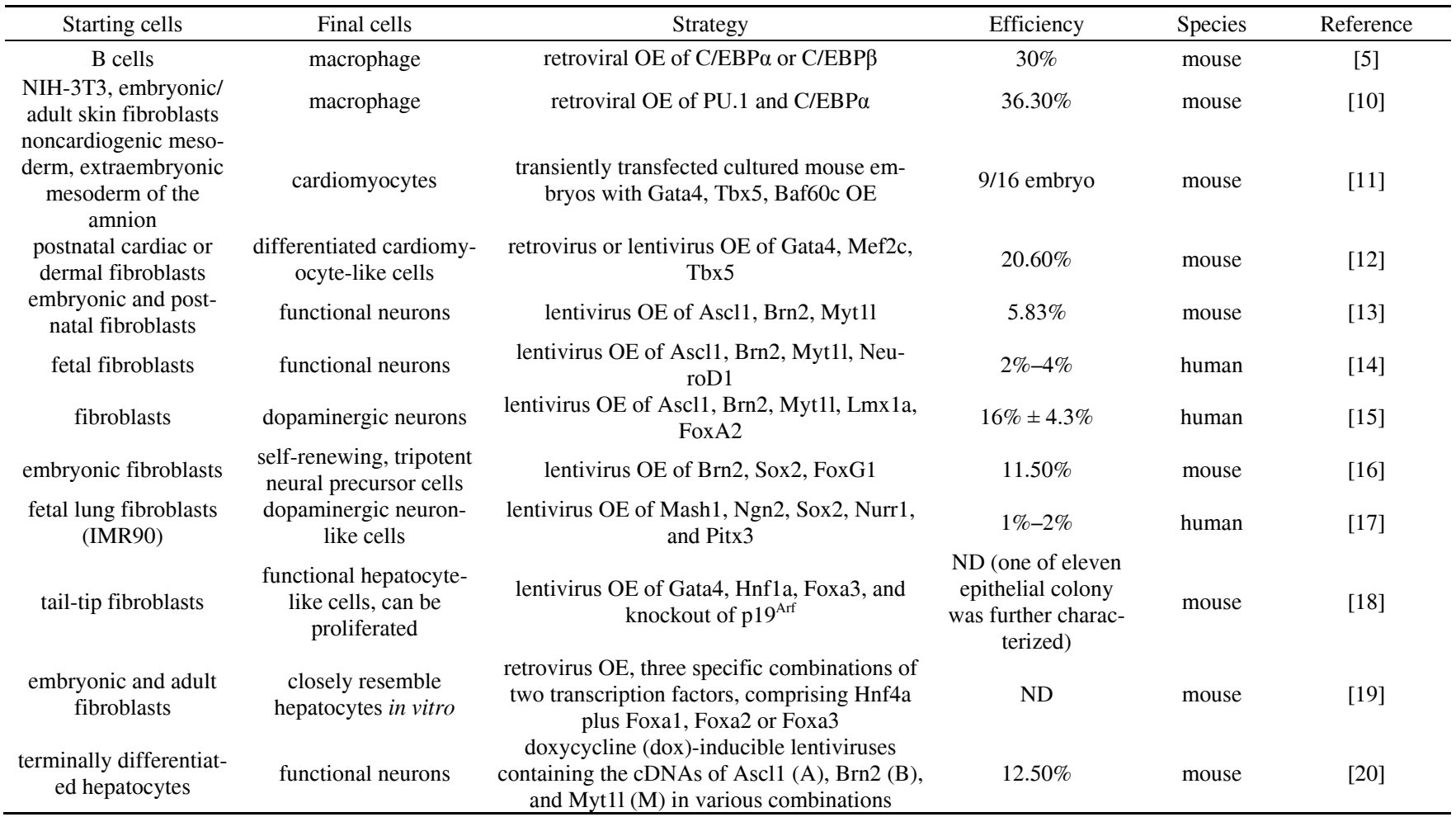

a) ND, not detected; OE, overexpression. 


\section{Dedifferentiation}

Dedifferentiation is the reversion of differentiated cells to a less differentiated state with loss of specialized cell functions and structures, which can be differentiated into other cell types. Induced conversion of somatic cells to pluripotent stem cells (i.e., iPS cells) can be considered as the most typical example. The majority of dedifferentiation-based strategies of lineage reprogramming are cell conversions of mature cells to unipotent or multipotent stem cells, which has been achieved with blood, cardiac and neural cells as well as melanocytes (Table 2).

iPS reprogramming factors are mostly used to induce starting cells into an unstable intermediate population [25] that can be differentiated into target cells by external cues such as specific growth factors. The use and combinations of iPS reprogramming factors are not the same for distinct target cells. For example, forced expression of a single transcription factor, Oct-4, allows the generation of an intermediate population that gives rise to blood lineages [22], whereas at least three factors including Oct-4, which is indispensable, are required for cell conversion to cardiac and neural cells [24,25]. However, Their et al. [26] reported that constitutive induction of Sox2, Klf4, and c-Myc while strictly limiting Oct-4 activity to the initial phase of reprogramming allows the generation of stably expandable neural stem cells (NSCs). Because Sox2, Klf4, and c-Myc are endogenous transcription factors in NSCs, their expression is not limited during lineage reprogramming. Another study reported that transient induction of the four reprogramming factors (Oct-4, Sox2, Klf4, and c-Myc) could efficiently convert fibroblasts to functional neural stem/progenitor cells
[25]. These observations indicate that exogenous expression of iPS cell reprogramming factors must be controlled for the production of stably expandable target cells.

Interestingly, adult hematopoietic programs are activated in $\mathrm{CD}_{4} 5^{+}$fibroblasts overexpressing Oct-4 [22]. Erythropoietin-treated $\mathrm{CD}_{4} 5^{+}$fibroblasts overexpressing Oct-4 generate erythroid cells expressing adult $\beta$-globin protein rather than embryonic $\zeta$-globin [22] that is always activated in embryonic hematopoietic programs. Ectopic expression of Lin28 reprograms hematopoietic stem/progenitor cells obtained from adult bone marrow to mediate multilineage reconstitution that resembles fetal lymphopoiesis including increased development of B-1a, marginal zone B, gamma/delta $\mathrm{T}$ cells, and natural killer $\mathrm{T}$ cells [23]. Although these two studies are not comparable because of distinct species, starting cells, and transcription factors, it can be concluded that such a route of dedifferentiation may not be the only one.

It is noteworthy that both transdifferentiation and dedifferentiation strategies enable the expansion and/or self-renewal of target cells [16,18,25,26]. A typical example includes the work by Their et al. [26]. They generated neurosphere-like colonies that can be expanded for more than 50 passages, which may facilitate potential applications in regenerative medicine.

\section{Transdetermination}

Transdetermination refers to reprogramming of a committed but not yet fully differentiated cell type into another [7]. The adult stem cell plasticity of HSCs and MSCs can be catego-

Table 2 Dedifferentiation ${ }^{\mathrm{a}}$

\begin{tabular}{|c|c|c|c|c|c|}
\hline Starting cells & Final cells & Strategy & Efficiency & Species & Reference \\
\hline mature B cells & $\mathrm{T}$ cells & $\begin{array}{l}\text { by dedifferentiation to uncommitted progeni- } \\
\text { tors in conditional Pax } 5 \text { deletion mice model }\end{array}$ & ND & mouse & [21] \\
\hline $\begin{array}{l}\text { adult/postnatal skin } \\
\text { fibroblasts }\end{array}$ & $\begin{array}{c}\mathrm{CD} 45^{+} \text {hematopoietic } \\
\text { progenitors }\end{array}$ & $\begin{array}{l}\text { addition of hematopoietic growth factors } 21 \\
\text { days after lentivirus OE of Oct- } 4 \text { under iPS } \\
\text { conditions }\end{array}$ & $7.5 \%-56.1 \%$ & human & [22] \\
\hline $\begin{array}{l}\text { adult bone marrow } \\
\text { LSK }^{+} \mathrm{HS} / \mathrm{PCs}\end{array}$ & $\begin{array}{l}\text { accquired the ability of } \\
\text { fetal lymphopoiesis }\end{array}$ & retrovirus $\mathrm{OE}$ of $\operatorname{Lin} 28$ & ND & mouse & {$[23]$} \\
\hline embryonic fibroblasts & cardiomyocytes & $\begin{array}{l}\text { retrovirus or transient OE of Oct4, Sox } 2, \text { Klf4, } \\
\text { c-Myc (dispensable), adding JAK inhibitor and } \\
\text { BMP4 4-9 days after culture under iPS condi- } \\
\text { tions }\end{array}$ & $40 \%$ & mouse & [24] \\
\hline $\begin{array}{l}\text { embryonic fibroblasts, } \\
\text { adult tail-tip fibroblasts }\end{array}$ & $\begin{array}{l}\text { proliferating functional } \\
\text { neural stem/progenitor } \\
\text { cells }\end{array}$ & $\begin{array}{l}\text { transient OE of the four reprogramming factors } \\
\text { (Oct4, Sox } 2, \text { Klf4, and c-Myc), reprogrmming } \\
\text { for two steps ( } 4 \text { days under iPS condi- } \\
\text { tions+9-13 days with bFGF, EGF, FGF4) }\end{array}$ & $\begin{array}{l}\text { embryonic fibro- } \\
\text { blasts } \\
(0.5 \%-0.69 \%) \\
\text { adult tail-tip fibro- } \\
\text { blasts }(0.07 \%)\end{array}$ & mouse & [25] \\
\hline fibroblasts & $\begin{array}{l}\text { neurosphere-like colo- } \\
\text { nies, expanded for more } \\
\text { than } 50 \text { passages }\end{array}$ & $\begin{array}{c}\text { constitutively inducing Sox } 2, \mathrm{Klf} 4 \text {, and c-Myc } \\
\text { while strictly limiting Oct } 4 \text { activity to the initial } \\
\text { phase of reprogramming (curtailed } \\
\text { reprogramming) }\end{array}$ & ND & mouse & [26] \\
\hline $\begin{array}{l}\text { mature pigmented epi- } \\
\text { dermal-derived mela- } \\
\text { nocytes }\end{array}$ & $\begin{array}{l}\text { functional multipotent } \\
\text { neural crest (NC) } \\
\text { stem-like cells }\end{array}$ & $\begin{array}{l}\text { forced expression of the intracellular domain of } \\
\text { Notch1 (NIC1) alone }\end{array}$ & $30 \%$ & human & [27] \\
\hline
\end{tabular}

a) ND, not detected; OE, overexpression. 
rized under transdetermination. For example, Jang et al. [28] isolated a small Fr25lin ${ }^{-}$HSC population from adult mouse bone marrow. RT-PCR analysis demonstrated that hepatocyte-specific marker genes were induced at $48 \mathrm{~h}$ after indirect coculture of Fr25lin ${ }^{-}$populations with injured minced liver tissues, and coculture with normal liver tissues or culture alone were the controls. Our laboratory [29] previously isolated a subset of umbilical cord blood-derived $\beta_{2} \mathrm{~m}^{-} c$ $\mathrm{Met}^{+}$cells that display some morphological characteristics of hepatocytes after indirect coculture with transgenic hepatic stellate cells. Several hepatocyte-specific genes are also expressed at mRNA and protein levels. In addition, the induced cells display liver-specific functions. These results suggest that a subset of umbilical cord blood cells can be transdeterminated into hepatocyte-like cells under indirect coculture conditions. We also showed that approximately $70 \%$ of the MSCs from human umbilical cord blood exhibit a typical neuron-like phenotype in vitro when provided with suitable external clues [30].

In subsequent studies, researchers reported that cell fusion might be the principal mechanism of adult stem cell plasticity. Cell fusion is a cellular combination of donor blood cells with host cells to produce heterokaryons with a hybrid or mixed phenotype. Cell fusion has been confirmed in a liver transplantation model under normal and pathological conditions, which exhibits a frequency between $1 / 10^{4}$ and $1 / 10^{6}$. Wang et al. [31] and Vassilopoulos et al. [32] first detected these hybrid cells in $\mathrm{FAH}^{-1-}$ mice. It has been established that myelomonocytic cells are the major source of hepatocyte fusion partners. However, Tanabe et al. [33] detected hepatocyte-like cells with mouse and human chromosomes, and cells with only human chromosomes in receipt mice transplanted with human cord blood cells. These studies demonstrate that both cell fusion and transdetermination may be the mechanisms for human cord blood cell-derived hepatocyte-like cells in transplanted mouse models.

However, the limited knowledge of external cues in transdetermination hinders the clinical potential of regenerative medicine, resulting in the low frequency of emerging target cells after growth factor induction in vitro or transplantation into an animal model of injury in vivo. Therefore, we have performed genetic manipulation of adult stem cells by a transcription factor-based transdetermination strategy. For example, such an approach can promote neural differentiation of human bone marrow MSCs by overexpression of brain-derived neurotrophic factor [34] or knockdown of neuron-restrictive silencer factor (NRSF) [35]. Moreover, pancreatic differentiation is enhanced by overexpression of pancreatic and duodenal homeobox 1 in human bone marrow MSCs [36] or knockdown of NRSF in human amniotic fluid-derived stem cells [37]. Interestingly, the same genetic modification with one transcription factor (NRSF) will trigger distinct cell conversion in human bone marrow MSCs and human amniotic fluid-derived stem cells, sug- gesting that the developmental stage of starting cells is involved in determination of cell reprogramming.

\section{Physiological and pathogenic cell conversions}

Metaplasia refers to more general transformation of one tissue type into another, which is usually stimulated by chronic inflammation $[7,38]$. It often involves the transformation of undifferentiated stem or progenitor cells into another cell type that is similar in nature. Common examples of metaplasia include squamous, intestinal and mesenchymal metaplasia.

Another common example of cell conversion is epithelial to mesenchymal transition (EMT). It is activated by the expression of important inducers of EMT, including transcription factors Snail, Slug and Twist in epithelial cells, resulting in changes of the cellular behavior. During the EMT process, the expression of E-cadherin and other epithelial cell markers is downregulated, while the expression of mesenchymal cell markers including $\mathrm{N}$-cadherin, vimentin and $\alpha$-smooth muscle actin is upregulated. The epithelial cells adopt a mesenchymal cell phenotype in appearance and acquire motility as well as invasive properties. A reversal of EMT process is mesenchymal-epithelial transition (i.e., MET). EMT-MET plasticity plays an important role in embryonic development, iPS cell reprogramming, and tumor progression [39-41]. It is certain that new physiological and pathogenic cell conversions will be discovered with the development of lineage tracing technology. The differences between the several types of cell conversions mentioned above are listed in Figure 1.

\section{Application prospects of lineage reprogram- ming}

Thus far, the strategy for lineage reprogramming has predominantly been forced expression of transcription factors. However, cell conversion from fibroblasts to neural cells has been reported by ectopic expression of microRNAs including miR-9/9* and miR-124 [42], but at a low rate of conversion. This process is facilitated by NeuroD2 overexpression and the addition of neurogenic transcription factors Ascl1 and Myt11. It is unknown whether the effects of interactions in the network of miRNA-target genes on reprogramming can be controlled or predicted.

In terms of application, the use of small molecules may be a good choice, which will facilitate the emergence of clinical applications. For example, during dedifferentiation, the addition of a JAK inhibitor can inhibit long-term expression of exogenous transcription factors including Oct-4, Sox2, Klf4 and c-Myc, which will block further dedifferentiation from an unstable intermediate to an iPS cell [24]. Small molecules have an enormous potential to facilitate the 


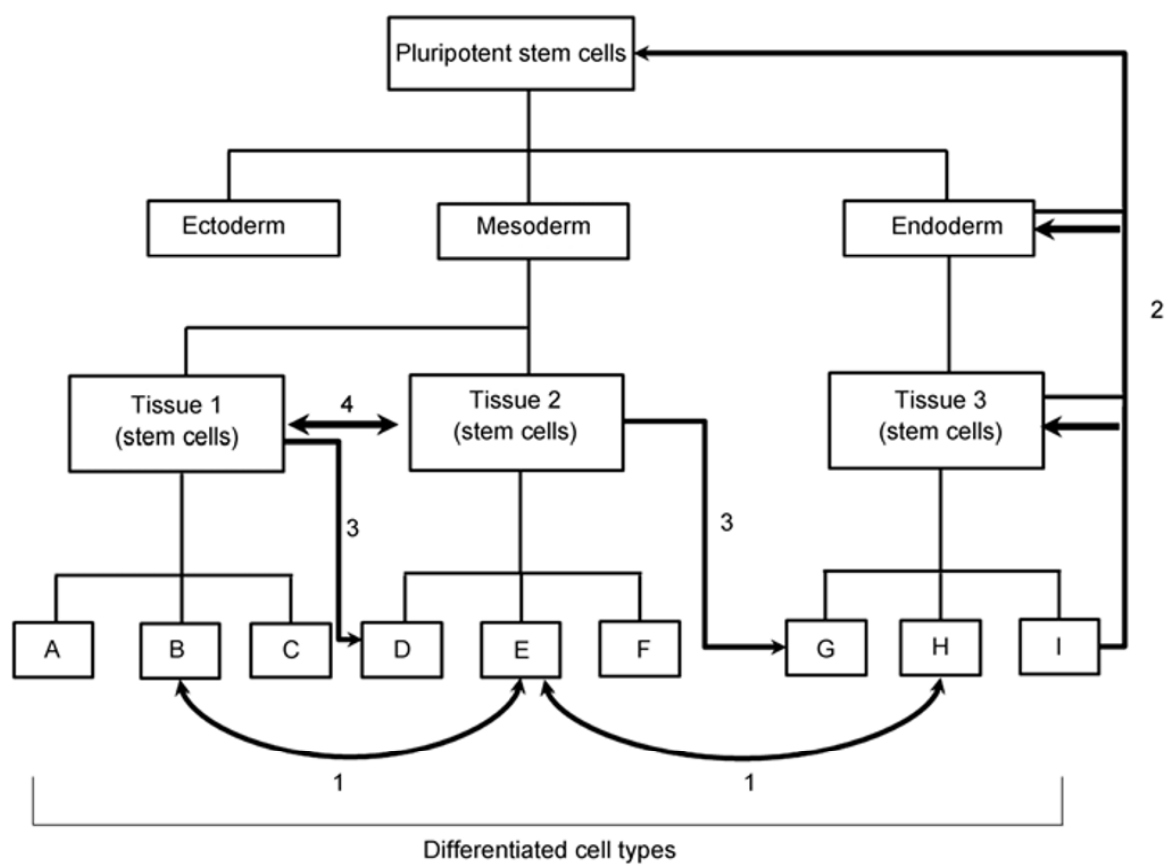

Figure 1 A simplified schematic of lineage reprogramming strategies. 1, transdifferentiation; 2, dedifferentiation; 3, transdetermination; 4, metaplasia. Transdifferentiation is cell conversion between differentiated cells originating from the same or different germ layers. In dedifferentiation, cells are converted into stem cells at different developmental stages in addition to pluripotent stem cells. The starting cells can also be stem cells (except pluripotent stem cells) in addition to differentiated cells. Transdifferentiation can also occur between the same or different germ layers. Metaplasia is transformation between tissue-specific stem cells under pathological conditions.

application of lineage reprogramming. Thus far, a growing number of small molecules have proved to be efficient and safe in reprogramming [43]. Perhaps several lineagereprogramming cocktails of small molecules suitable for different target cells will be developed in the near future. Genetic manipulation involves overexpression, knockdown [18] or knockout [21] strategies. In most studies, transfection of transcription factors uses viral vectors that have safety concerns, as is the case with iPS cells. Thus, it is of crucial importance to reprogramme fibroblasts to NSCs by transient overexpression of Oct-4, Sox2, Klf4 and c-Myc [25], and mouse mesoderm to heart tissues by transient overexpression of Gata4, Tbx5, and Baf60c [11]. Transient expression of lineage reprogramming factors to achieve a full phenotypic switch is one of the most promising strategies in stem cell and regenerative medicine translational research.

A comparison of the lineage and iPS cell reprogramming strategies is listed in Table 3. In Table 3, limited cell scaling is in contrast to the near unlimited capacity for expansion of iPS/embryonic stem cells. In fact, lineage reprogramming-derived expandable NSCs and proliferating hepatocytes have been achieved recently $[18,25,26]$. In addition, in vivo regeneration has been described in a report by Zhou et al. [45]. Adenoviral vectors encoding a specific combination of three transcription factors, Ngn3, Pdx1 and Mafa, were delivered into the differentiated pancreatic exocrine cells of adult mice of which $2 / 3$ were infected successfully after one month. About $20 \%$ of the infected cells were reprogrammed into insulin-secreting cells that closely resembled $\beta$-cells. Such an approach can be considered as in vivo lineage reprogramming and a new type of gene therapy. In terms of the tumor risk, it is low for lineage reprogramming without reversion to a pluripotent stem cell state. Notably, it is important to precisely control the time point when unstable intermediates in dedifferentiation strategy are emerging. Further research is still needed to elucidate new transcription factors, cell conversion strategies, and the underlying mechanisms of lineage reprogramming.

In summary, compared with studies of adult stem cell plasticity, lineage-reprogramming research has made great progress. Specifically, the cell conversion efficiency has been greatly increased, and reprogrammed cells can be expanded. These achievements establish the clinical foundation for lineage reprogramming in regenerative medicine.

In the near future, for a bedridden patient, it may be routine to take a skin sample to culture fibroblasts under standard conditions, and a reprogramming kit will be used to reprogramme the fibroblasts into expandable stem/progenitor cells that will be injected to regenerate the injured tissues of the patient. Perhaps this procedure will become routine work for a department of stem cell and regenerative medicine in a hospital. The customized and individualized clinical applications of reprogrammed stem/progenitor cells are the most attractive to researchers and patients in regenerative medicine. 
This work was supported by the National High Technology Research and Development Program of China (2011AA020109) and the National Basic Research Program of China (2011CB964804).

1 Yamanaka S. Strategies and new developments in the generation of patient-specific pluripotent stem cells. Cell Stem Cell, 2007, 1: 39-49

2 Zhou Q, Melton D A. Extreme makeover: converting one cell into another. Cell Stem Cell, 2008, 3: 382-388

3 Takahashi K, Yamanaka S. Induction of pluripotent stem cells from mouse embryonic and adult fibroblast cultures by defined factors. Cell, 2006, 126: 663-676

4 Davis R L, Weintraub H, Lassar A B. Expression of a single transfected cDNA converts fibroblasts to myoblasts. Cell, 1987, 51: 987-1000

5 Xie H, Ye M, Feng R, et al. Stepwise reprogramming of B cells into macrophages. Cell, 2004, 117: 663-676

6 Petersen B E, Bowen W C, Patrene K D, et al. Bone marrow as a potential source of hepatic oval cells. Science, 1999, 284: 1168-1170

7 Graf T, Enver T. Forcing cells to change lineages. Nature, 2009, 62: 587-594

8 Jiang Y, Jahagirdar B N, Reinhardt R L, et al. Pluripotency of mesenchymal stem cells derived from adult marrow. Nature, 2002, 418: 41-49

9 Krause D S, Theise N D, Collector M I, et al. Multi-organ, multi-lineage engraftment by a single bone marrow-derived stem cell. Cell, 2001, 105: 369-377

10 Feng R, Desbordes S C, Xie H, et al. PU.1 and C/EBPalpha/beta convert fibroblasts into macrophage-like cells. Proc Natl Acad Sci USA, 2008, 105: 6057-6062

11 Takeuchi J K, Bruneau B G. Directed transdifferentiation of mouse mesoderm to heart tissue by defined factors. Nature, 2009, 459: 708-711

12 Ieda M, Fu J D, Delgado-Olguin P, et al. Direct reprogramming of fibroblasts into functional cardiomyocytes by defined factors. Cell, 2010, 142: 375-386

13 Vierbuchen T, Ostermeier A, Pang Z P, et al. Direct conversion of fibroblasts to functional neurons by defined factors. Nature, 2010, 463 : 1035-1041

14 Pang Z P, Yang N, Vierbuchen T, et al. Induction of human neuronal cells by defined transcription factors. Nature, 2011, 476: 220-223

15 Pfisterer U, Kirkeby A, Torper O, et al. Direct conversion of human fibroblasts to dopaminergic neurons. Proc Natl Acad Sci USA, 2011, 108: 10343-10348

16 Lujan E, Chanda S, Ahlenius H, et al. Direct conversion of mouse fibroblasts to self-renewing, tripotent neural precursor cells. Proc Natl Acad Sci USA, 2012, 109: 2527-2532

17 Liu X, Li F, Stubblefield E A, et al. Direct reprogramming of human fibroblasts into dopaminergic neuron-like cells. Cell Res, 2012, 22: 321-332

18 Huang $\mathrm{P}, \mathrm{He} \mathrm{Z}$, Ji S, et al. Induction of functional hepatocyte-like cells from mouse fibroblasts by defined factors. Nature, 2011, 475: 386-389

19 Sekiya S, Suzuki A. Direct conversion of mouse fibroblasts to hepatocyte-like cells by defined factors. Nature, 2011, 475: 390-393

20 Marro S, Pang Z P, Yang N, et al. Direct lineage conversion of terminally differentiated hepatocytes to functional neurons. Cell Stem Cell, 2011, 9: 374-382

21 Cobaleda C, Jochum W, Busslinger M. Conversion of mature B cells into $\mathrm{T}$ cells by dedifferentiation to uncommitted progenitors. Nature, 2007, 449: 473-477

22 Szabo E, Rampalli S, Risueño R M, et al. Direct conversion of human fibroblasts to multilineage blood progenitors. Nature, 2010, 468: $521-526$
23 Yuan J, Nguyen C K, Liu X, et al. Lin28b reprograms adult bone marrow hematopoietic progenitors to mediate fetal-like lymphopoiesis. Science, 2012, 335: 1195-1200

24 Efe J A, Hilcove S, Kim J, et al. Conversion of mouse fibroblasts into cardiomyocytes using a direct reprogramming strategy. Nat Cell Biol, 2011, 13: 215-222

25 Kim J, Efe J A, Zhu S, et al. Direct reprogramming of mouse fibroblasts to neural progenitors. Proc Natl Acad Sci USA, 2011, 108: 7838-7843

26 Thier M, Wörsdörfer P, Lakes Y B, et al. Direct conversion of fibroblasts into stably expandable neural stem cells. Cell Stem Cell, 2012, 10: 473-479

27 Zabierowski S E, Baubet V, Himes B, et al. Direct reprogramming of melanocytes to neural crest stem-like cells by one defined factor. Stem Cells, 2011, 29: 1752-1762

28 Jang Y, Collector M I, Baylin S B, et al. Hematopoietic stem cells convert into liver cells within days without fusion. Nature Cell Biol, 2004, 6: 532-539

29 Wang Y, Nan X, Li Y, et al. Induction of umbilical cord blood-derived $\beta 2 \mathrm{~m}-\mathrm{c}-\mathrm{Met}+$ cells into hepatocyte-like cells by coculture with CFSC/HGF cells. Liver Transpl, 2005, 11: 635-643

30 Hou L, Cao H, Wang D, et al. Induction of umbilical cord blood mesenchymal stem cells into neuron-like cells in vitro. Int J Hematol, 2003, 78: 256-261

31 Wang X, Willenberg H, Akkari Y, et al. Cell fusion is the principal source of bone marrow derived hepatocytes. Nature, 2003, 422: 897-901

32 Vassilopoulos G, Wang P, Russell D W. Transplanted bone marrow regenerates liver by cell fusion. Nature, 2003, 422: 901-904

33 Tanabe Y, Tajima F, Nakamura Y, et al. Analyses to clarify rich fractions in hepatic progenitor cells from human umbilical cord blood and cell fusion. Biochem Biophys Res Commun, 2004, 324: 711-718

34 Zhao L X, Zhang J, Cao F, et al. Modification of the brain-derived neurotrophic factor gene: a portal to transform mesenchymal stem cells into advantageous engineering cells for neuroregeneration and neuroprotection. Exp Neurol, 2004, 190: 396-406

35 Yang Y, Li Y, Lv Y, et al. NRSF silencing induces neuronal differentiation of human mesenchymal stem cells. Exp Cell Res, 2008, 314: 2257-2265

36 Li Y, Zhang R, Qiao H, et al. Generation of insulin-producing cells from PDX-1 gene-modified human mesenchymal stem cells. J Cell Physiol, 2007, 211: 36-44

37 Li B, Wang S, Liu H, et al. Neuronal restrictive silencing factor silencing induces human amniotic fluid-derived stem cells differentiation into insulin-producing cells. Stem Cells Dev, 2011, 20: 1223-1231

38 Slack J M. Metaplasia and transdifferentiation: from pure biology to the clinic. Nat Rev Mol Cell Biol, 2007, 8: 369-378

39 Ouyang G, Wang Z, Fang X, et al. Molecular signaling of the epithelial to mesenchymal transition in generating and maintaining cancer stem cells. Cell Mol Life Sci, 2010, 67: 2605-2618

40 Thiery J P, Acloque H, Huang R Y, et al. Epithelial-mesenchymal transitions in development and disease. Cell, 2009, 139: 871-890

41 Abollo-Jiménez F, Jiménez R, Cobaleda C. Physiological cellular reprogramming and cancer. Semin Cancer Biol, 2010, 20: 98-106

42 Yoo A S, Sun A X, Li L, et al. MicroRNA-mediated conversion of human fibroblasts to neurons. Nature, 2011, 476: 228-231

43 Li W, Jiang K, Ding S. Concise review: A chemical approach to control cell fate and function. Stem Cells, 2012, 30: 61-68

44 Vierbuchen T, Wernig M. Direct lineage conversions: unnatural but useful? Nat Biotechnol, 2011, 29: 892-907

45 Zhou Q, Brown J, Kanarek A, et al. In vivo reprogramming of adult pancreatic exocrine cells to beta-cells. Nature, 2008, 455: 627-632

Open Access This article is distributed under the terms of the Creative Commons Attribution License which permits any use, distribution, and reproduction in any medium, provided the original author(s) and source are credited. 